\title{
KELEA Activated Water as an Alternative to Stem Cell Injections in Regenerative Medicine
}

\begin{abstract}
The use of stem cells in regenerative medicine was initially promoted as a means of treating illnesses due to the functional lack of specific types of specialized cells. Stem cells were further seen as a way to i) replace defective organs with better performing organs, such as the heart, ii) restore diminished brain functions, iii) replace lost limbs, and iv) boost the body's defenses against both cancer and infectious diseases. The early reliance on using primitive embryonic cells for stem cell therapy was offset by showing that differentiated cells from an adult could be genetically reprogrammed to become replicating progenitor cells, which could then either naturally or genetically be transformed into multiple types of specialized cells. The clinical application of cell modifying technologies has been relatively slow, however, mainly due to lingering safety concerns regarding the possibility of the cells forming cancers. There are fewer safety concerns with the use of cells from umbilical cord blood (UCB) and from adipose tissue, which have retained the capacity to mature into different cell types. These mesenchymal stem cells (MSC) are currently being used in the empirical treatment of multiple clinical conditions. Their mode of action, however, is not that of replacing absent or diseased cells in the body, but rather it is the release of compounds able to i) suppress inflammation and ii) promote the regeneration of cells already existing within the body. An alternative cellular energy (ACE) pathway has been described as a source of cellular energy that is different from the energy obtained from food metabolism. It is expressed as an added dynamic or kinetic quality of the body's fluids. It results from the absorption of an external energy termed KELEA (kinetic energy limiting electrostatic attraction). The ACE pathway can be enhanced using KELEA activated water products. One such waterceutical ${ }^{\mathrm{TM}}$, called Enercel ${ }^{\mathrm{TM}}$, was originally regarded as a homeopathic remedy. In preliminary clinical studies, Enercel is showing similar benefits as those ascribed to the injection of MSC or of their derived compounds. Waterceutical products, such as Enercel, are simpler, safer and more effective potential alternatives to the in vivo injections of MSC or MSC-derived compounds. An additional advantage is that certain KELEA activated fluids can support the ACE pathway, even when placed at or near the skin surface. Further clinical benefits can be expected when waterceuticals are used in conjunction with other means of enhancing the body's ACE pathway. Some of these additional therapeutic methods are discussed in this paper.
\end{abstract}

Keywords: Enercel ${ }^{\mathrm{TM}}$, enerceutical ${ }^{\mathrm{TM}}$, waterceutical ${ }^{\mathrm{TM}}$, stem, KELEA, ACE, iPSC
Volume II Issue 5 - 2018

\author{
W John Martin \\ University of Quantum Dynamics, USA
}

Correspondence: $W$ John Martin, University of Quantum Dynamics, 1634 Spruce Street, South Pasadena, CA, USA, Email wjohnmartin@ccid.org

Received: August 30,2018| Published: September 10,2018
Abbreviations: HSC, hematopoietic stem cells; ESC, embryonic stem cells; UCB, umbilical cord blood; MSC, mesenchymal stem cells; SVF, stromal vascular fraction; iPSC, induced pluripotent stem cells; PRP, platelet rich plasma; ACE, alternative cellular energy; KELEA, kinetic energy limiting electrostatic attraction; CSC, coppersilver-citrate

\section{Introduction}

The original intent of stem cell therapies was to provide patients with undifferentiated cells that could differentiate into the specific types of specialized cells, which were either absent or dysfunctional in the patients. ${ }^{1}$ Basically, the stem cells were intended to replenish the body with a fresh source of specific types of normally functioning cells. This goal clearly applied to the grafting of bone marrow cells into leukemia patients after the patients received radiation or chemotherapy. ${ }^{2}$ Ideally, the donors of the bone marrow derived hematopoietic stem cells (HSC) are genetically closely related to the individual recipients of the donor cells to minimize possible graft-versus-host and host-versusgraft immunological reactions. ${ }^{3}$ Replicating cells obtained from very early embryos are naturally destined to generate progeny cells that can collectively differentiate into all types of specialized cells., ${ }^{4,5}$ These very primitive replicating cells, however, retain the potential of developing into a teratoma and other tumors. There is a requirement, therefore, to somewhat genetically restrict these cells before they are clinically used as pluripotent embryonic stem cells (ESC). ${ }^{6}$ There is also an ethical issue in sacrificing human embryos as the source of therapeutic cells. This latter concern has been avoided by showing that cellular differentiation is a reversible process. Thus, mature cells obtained from an adult can be induced by genetic reprogramming into a more primitive or embryonic cell type. ${ }^{7}$ As with ESC, these induced pluripotent stem cells (iPSC), also have to be genetically restricted from potentially becoming tumors. Methods for reliably achieving this safety goal for primitive stem cells are not convincingly proven and this has delayed progress in the common use of iPSC. This barrier has not, however, prevented the extensive use of therapeutic cells other than ESC and iPSC.

\section{Sources of less primitive stem cells}

The focus of stem cell research has largely switched to the use of developing cells, which have undergone sufficient maturation to not be 
a threat of developing into tumors. Such developing cells can still retain the progenitor cell capacities of both replicating and transforming into more highly specialized cells. In addition to HSC, bone marrow also contains progenitor cells able to differentiate in tissue cultures into non-hematopoietic cells. ${ }^{8}$ Umbilical cord blood (UCB)from newborns similarly contain cells that can transform into a limited variety of nonhematopoietic cell types. ${ }^{9}$ Related multipotential, progenitor cells can also be derived from the amnion ${ }^{10}$ and the placenta. ${ }^{11}$ Although, the progeny cell types obtainable from birth-derived progenitor cells are not as diverse as the cell types derived from ESC or iPSC, they are, nevertheless, still referred to as stem cells. Except in circumstances in which cord blood has been stored at birth for future possible use by the donor, the newborn-derived cells are histoincompatible with the host. In the absence of immunosuppression these cells would not be expected to indefinitely survive within the recipient.

Liposuction-harvested fat tissue is also a source of partially differentiated progenitor cells ${ }^{12-15}$ referred to as mesenchymal stem cells (MSC). These cells are capable of differentiating not only into fat cells (adipocytes) but also into several other cell types. MSC in liposuction-obtained fat tissue can be selectively concentrated by centrifugation into a stromal vascular fraction (SVF) and may be further modified in tissue culture. ${ }^{16-18}$ In most patients, however, the fat tissue is not fractionated or cultured, but is transferred directly into the site of disease and/or injected intravenously. Relocating fat cells between tissue sites has been performed by plastic surgeons for a number of years in various cosmetic procedures. ${ }^{19}$ The transferred cells can potentially survive since there is no histocompatibility barrier when the cells are transferred between fat tissue sites in the same individual. Even so, some cosmetic plastic surgeons commonly delay reinjecting the fat tissue (personal communication). In the intervening period, the cells are stored in a freezer leading to a loss of the cells' viability. Successful cosmetic results are seemingly still obtainable using non-viable cells. The actual demonstration of persisting fat tissue-derived MSC or of their progeny in tissues of the recipients is lacking. Moreover, the overall clinical results using MSC available from histoin compatiblenew born sand from histocompatible adult adipose tissue or bone marrow are generally comparable, with, if anything, better overall success occurring with UCB cells from newborn infants.

\section{Proliferation of stem cell clinics treating numerous illnesses}

It is estimated that there are now over 600 stem cell clinics within the United States and many additional centers elsewhere in the world. These centers primarily offer UCB cells or adipose tissue-derived MSC as therapy for a wide variety of conditions. These include improving facial appearance, hair growth, traumatic joint damage, rheumatoid and osteoarthritis, spinal cord injuries, acute burns, autoimmune diseases, autism, pain syndromes, neurodegenerative illnesses, and cancer. ${ }^{20-30}$ Even relatively simple procedures are typically priced in excess of $\$ 10,000$, with no current coverage by medical insurance. Unfortunately, such commercial gains have dulled much of the critical reasoning regarding the use of stem cells and have raised questions regarding the objective assessments of clinical outcomes. In particular reports of fat embolism appear to be underplayed, ${ }^{31}$ as well as blindness from intraocular fat cell injections. ${ }^{32}$ It has also been suggested that some types of MSC can actually promote the growth of nascent tumor cells. ${ }^{33-35}$
Also entering into the commercial arena are dietary supplements, which are promoted as being especially nourishing for stem cells or as being able to better mobilize stem cells for delivery to damaged tissues. ${ }^{36}$ Several of these supplements comprise the same or similar herbal products, which were previously marketed as anti-oxidants. The anti-oxidant activity is an indirect indication that the products have available regions of separated electrical charges and are, therefore, dipolar. The significance of this statement is addressed below.

\section{Biological activity of factors released by stem cells}

As stated above, there is no evidence of long-term persistence of either UCB cells or fat-derived MSC in the recipients. This is especially apparent when the cells are inoculated into peripheral tissues, such as joints. Although, not widely discussed, the more reasonable view is that the injected cells release a variety of compounds that can seemingly assist in suppressing in suppressing symptoms and in repairing locally damaged tissues. These compounds or factors apparently act directly on the body's preexisting cells in a process referred to as a paracrine effect. ${ }^{37}$ The paracrine mode of action is fundamentally different from what was initially viewed as the primary mode of action of stem cells. Rather, the apparent benefits of inoculated stem cells are more directly comparable to the earlier uses of fetal tissue extracts as a rejuvenating therapy. ${ }^{38} \mathrm{~A}$ number of sub cellular components released from cultured stem cells (secretome) have been characterized. They can be in the form of cell-derived particles called exomes, ${ }^{39,40}$ or comprise soluble mediators, including cytokines. ${ }^{41}$ These materials can be directly injected into patients as alternatives to using viable cells. There is overlap in the biological benefits of compounds derived from stem cells with those reported as occurring with compounds present in autologous platelet rich plasma $(\mathrm{PRP})^{42,43}$ and with on-autologous extracts from amniotic fluids. ${ }^{44,45}$ The major biological functions of these various compounds are typically described asi) suppressing tissue damaging inflammation, and/orii) promoting the growth of regenerating normal tissues. These two actions are similarly achievable via the proposed paracrine effects of inoculated stem cells, without the cells becoming engrafted into the recipient's tissues.

\section{Alternative Cellular Energy (ACE) pathway}

Without the fanfare of stem cell therapy, there are several studies indicating that disease suppression and tissue regeneration can be achieved using energy-based therapies. The beneficial effects of these therapies can be explained in terms of the body having an alternative cellular energy (ACE) pathway. Thus, I have proposed that this pathway is a type of cellular energy, which is fundamentally different from the cellular energy obtained from the metabolism of food. ${ }^{46-56}$ The ACE pathway is reflected in an added dynamic (kinetic) quality of the body's fluids. It arises from an external force termed KELEA (kinetic energy limiting electrostatic attraction), which can enter into the body using three basic mechanisms. The first is assumed to be a natural antenna function of the oscillating electrical activity of the brain and possibly muscles, including the heart. ${ }^{57}$ Certain modes of the brain's electrical oscillations are likely to be more efficient than others, presumably with some level of conscious regulation. Second; this proposed human antenna function can also be enhanced by increasing the environmental levels of KELEA to which the individual is exposed ${ }^{58,59}$ This is achievable using devices that are driven by either oscillating electrical currents or by converging electromagnetic 
radiations, including light. The third and best documented method of enhancing the ACE pathway is the use of KELEA activated water or water activating compounds. Water and other fluids can be reliably activated using various dipolar compounds that are capable of attracting KELEA into fluids. ${ }^{60-64}$ Once the water is sufficiently activated, the activating compounds, referred to as enerceuticals ${ }^{\mathrm{TM}}$, can be removed by repeated dilutions, filtration, decanting or other means. KELEA activated water can also be produced by sufficient exposure to an increased environmental level of KELEA as created using various devices. The natural water in certain locations in the world is intrinsically activated. ${ }^{46}$ Depending upon how it is prepared and stored, the levels of KELEA activation can range from being minimal to being substantial. Highly activated water will retain its activation over a long period time if stored in a sealed container. Moreover, it can attract additional KELEA, which is transferrable to ordinary water that is placed close to the highly activated water.

\section{Clinical testing of KELEA activated water (Waterceuticals)}

A well-tested water ceutical product that is suitable for direct injection into humans is presently available. It is called Enercel and produced by World Health Advanced Technology, with its head office in Sarasota, FL. It was initially characterized as a homeopathic remedy related to an Argentinian product called HANSI (homeopathic activator of the natural system immune). The activating enerceuticals mainly comprise small amounts of various herbal tinctures plus some minerals. The concentrations of these compounds are further reduced by a factor of 1:10,000 using repeated 10-fold dilutions, each dilution being followed by succussion (jolting). Succussion is also used prior to the administration of Enercel formulations, which are available for intramuscular, intravenous, intraarticular, intranasal and/ or inhalational administration. A slightly different formulation is also available for direct topical use on the skin.

Intravenous administered Enercel reduces HIV levels in AIDS patients. ${ }^{65}$ There is an accompanying markedly improved sense of wellbeing. Inhalation of Enercel, as an additional therapy, cleared mycobacteria from the sputum in tuberculosis infected AIDS patients. ${ }^{65}$ Enercel can also lead to the painless regression of cancers, presumably by providing sufficient cellular energy for tumor cell apoptosis. ${ }^{66,67}$ The severity of tropical diarrhea in children was significantly lessened using two intramuscular injections of Enercel. ${ }^{68}$

Enercel and another KELEA activated waterceutical prepared using copper-silver-citrate (CSC) complexes were also remarkably successful in achieving one or more of the following: Scar-free healing of acute burns; regeneration of ischemic limb tissues; healing of infected wounds; and the repair of a traumatic penetrating injury. ${ }^{69}$ Intraperitoneal administration of the CSC solution dramatically prevented surgical adhesions from forming after a cesarean section was performed in a goat, with additional overall improvement in the health of the animal. ${ }^{69}$ Following intraarticular injections, relatively small amounts $(<1 \mathrm{ml})$ of another waterceutical product promptly relieved arthritic pain in several treated humans and animals (unpublished).

\section{Overlapping benefits achieved by stem cell therapy and KELEA activated water}

A reasonable inference from these observations is that many of the current stem cell-based therapies will likely be equally if not more achievable through the direct administration of pharmaceutical grade KELEA activated solutions. One area for directly comparing the benefits of stem cells with waterceutical products is in the treatment of acute burns. Enercel has completely prevented scar tissue from forming at a burn site when it was applied shortly after a second to third-degree burn. A major advantage of waterceuticals for parental use is that essentially all of the water activating compounds can be removed prior to their administration. Depending upon the type of enerceuticals used, this can be achieved by repeated dilutions, decanting, or zero-residue filtration. The absence of detectable compounds in the water will help avoid many of the FDA regulatory requirements that are currently associated with the use of stem cells and/or stem cell products. ${ }^{70-78}$ There should be even less regulatory concerns with the use of water activating devices that function by increasing the available environmental levels of KELEA.

Formal clinical research studies will require the direct comparison of KELEA related fluids with regular pharmaceutical grade fluids, administered in the absence of any additional treatments. For more routine care, a combination of various ACE-pathway enhancing methods can be utilized. A sustainable improvement in the ACE pathway is potentially achievable through efforts to optimize the proposed KELEA antenna function of the brain through appropriate training and education. Efficient food metabolism can also be addressed. It is interesting to note that some of the major stem cell clinics are also incorporating positive psychology, nutrition and lifestyle changes into their programs. This approach follows the suggestion that the stem cells are analogous to seeds, but that they still require a healthy soil in the form of an improved body's terrain to thrive. This type of reasoning falsely assumes long-term persistence of the stem cells. A simpler explanation is that the diet and lifestyle changes are conducive to a better ACE pathway.

\section{Conclusion}

The current wave of interest in mesenchymal stem cell (MSC) therapy may lead to a better understanding of the alternative cellular energy (ACE) pathway. This is especially relevant since the primary beneficial effects of MSC and their products may well be mediated via the ACE pathway. The premise of this article is that injections of pharmaceutical grade waterceuticals will achieve similar if not superior benefits to those occurring through the use of MSC. Enercel is a clinically tested waterceutical for parental administration. It can be directly compared with injected MSC in the therapy of diverse clinical illnesses. If the outcomes are comparable, the use of Enerce land other waterceuticals will be far less expensive than using stem cells and will certainly face fewer regulatory burdens. Waterceuticals are also suitable for regular ingestion, inhalation or direct application to the skin. The practical clinical uses of waterceuticals can logically be coupled with other therapeutic approaches to enhance the ACE pathway.

\section{Acknowledgements}

The University of Quantum Dynamics (UQD) is a coalition of entities, including the Institute of Progressive Medicine, a component of MI Hope Inc., a non-profit public charity. UQD is dedicated to establishing a role for energy-based medicine in routine medical care, including patient directed self care. UQD current programs include REACH (Regenerative Energy Assisted Clinical TM). The author is particularly thankful to Dr. David Christner, CEO of World Health Advanced Technologies, for providing unpublished clinical data on 
Enercel and for many fruitful discussions.

\section{Conflict of interest}

The author declares no conflict of interest.

\section{References}

1. Sampogna G, Guraya SY, Forgione A. Regenerative medicine: Historical roots and potential strategies in modern medicine. Journal of Microscopy and Ultra structure. 2015;3(3):101-107.

2. Rowe JM, Lazarus HM. Genetically haploidentical stem cell transplantation for acute leukemia. Bone Marrow Transplantation. 2001;27(7):669-676.

3. Petersdorf EW, Gooley T, Anasetti C, et al. Optimizing outcome after unrelated marrow transplantation by comprehensive matching of HLA class I and II alleles in the donor and recipient. Blood. 1998;92(10):35153520 .

4. Hoffman LM, Carpenter MK. Characterization and culture of human embryonic stem cells. Nature Biotechnology. 2005;23(6):699-708.

5. Thomson JA, Itskovitz-Eldor J, Shapiro SS, et al. Embryonic stem cell lines derived from human blastocysts. Science. 1998;282(5391):11451147.

6. Audet J. Stem cell bioengineering for regenerative medicine. Expert Opin Biol Ther. 2004;4(5):631-644.

7. Shtrichman R, Germanguz I, Itskovitz-Eldor J. Induced pluripotent stem cells (iPSCs) derived from different cell sources and their potential for regenerative and personalized medicine. Curr Mol Med. 2013;13(5):792805 .

8. Pontikoglou C, Deschaseaux F, Sensebe L, et al. Bone marrow mesenchymal stem cells:biological properties and their role in hematopoiesis and hematopoietic stem cell transplantation. Stem Cell Rev. 2011;7(3):569-589.

9. El Omar R, Beroud J, Stoltz JF, et al. Umbilical cord mesenchymal stem cells:the new gold standard for mesenchymal stem cell-based therapies? Tissue Eng Part B Rev. 2014;20(5):523-544.

10. Miki T. Stem cell characteristics and the therapeutic potential of amniotic epithelial cells. Am J Reprod Immunol. 2018;29:e13003.

11. Malek A, Bersinger NA. Human placental stem cells: biomedical potential and clinical relevance. J Stem Cells. 2011;6(2):75-92.

12. Frese L, Dijkman PE, Hoerstrup SP. Adipose tissue-derived stem cells in regenerative medicine. Transfusion Medicine and Hemotherapy. 2016;43(4):268-274.

13. Bateman ME, Strong AL, Gimble JM, et al. Concise review: Using fat to fight disease: A systematic review of non homologous adipose-derived stromal/stem cell therapies. Stem Cells. 2018;36(9):1311-1328.

14. Zuk P A, Zhu M, Ashjian P, et al. Human adipose tissue is a source of multipotent stem cells. Molecular Biology of the Cell. 2002;13(12):42794295.

15. Alexandrer RW, Harrell DB. Autologous fat grafting: use of closed syringe microcannula system for enhanced autologous structural grafting. Clin Cosmet Investig Dermatol. 2013;6:91-102

16. Cai W, Yu LD, Tang X, et al. The stromal vascular fraction improves maintenance of the fat graft volume: A systematic review. Ann Plast Surg. 2018;81(3):367-371.

17. Paz Rodriguez J, Murphy M, Hong S, et al. Autologous stromal vascular fraction therapy for rheumatoid arthritis: Rationale and clinical safety. Int Arch Med. 2012;5:5
18. Riordan N, Ichim T, Min W, et al. Non-expanded adipose stromal vascular fraction cell therapy for multiple sclerosis. J Transl Med. 2009;7:29

19. De Pedroza LV. Fat transplantation to the buttocks and legs for aesthetic enhancement or correction of deformities:long-term results of large volumes of fat transplant. Dermatol Surg. 2000;26(12):1145-1149.

20. Zarei F, Abbaszadeh A. Stem cell and skin rejuvenation. J Cosmet Laser Ther. 2018;20(3):193-197.

21. Anderi R, Makdissy N, Azar A, et al. Cellular therapy with human autologous adipose-derived adult cells of stromal vascular fraction for alopecia areata. Stem Cell Res Ther. 2018;9(1):141.

22. Keerthi N, Chimutengwende-Gordon M, Sanghani A, et al. The potential of stem cell therapy for osteoarthritis and rheumatoid arthritis. Curr Stem Cell Res Ther. 2013;8(6):444-450.

23. Chhabra HS. Stem cell therapy in spinal cord injuries:current concepts. $J$ Assoc Physicians India. 2012;60 Suppl:30-33.

24. Li Z, Maitz P. Cell therapy for severe burn wound healing. Burns Trauma. 2018;6:13.

25. Cipriani P, Carubbi F, Liakouli V, et al. Stem cells in autoimmune diseases: Implications for pathogenesis and future trends in therapy. Autoimmun Rev. 2013;12(7):709-16.

26. Ichim T, Solano F, Glenn E, et al. Stem cell therapy for autism. $J$ Translational Medicine. 2007;5:30

27. Huh Y, Ji RR, Chen G. Neuroinflammation, bone marrow stem cells, and chronic pain. Front Immunol. 2017;8:1014.

28. Wang T, Shiqdar S, Gantier MP, et al. Cancer stem cell targeted therapy:progress amid controversies. Oncotarget. 2015;6(42):44191-206.

29. Mendes Filho D, Ribeiro PDC, Oliveira LF, et al. Therapy with mesenchymal stem cells in Parkinson disease: History and perspectives. Neurologist. 2018;23(4):141-147.

30. Kim SU, De Vellis J. Stem cell-based cell therapy in neurological diseases:a review. J Neurosci Res. 2009;87(10):2183-2200.

31. Ross RM, Johnson GW. Fat embolism after liposuction. Chest. 1988;93(6):1294-1295.

32. Kuriyan AE, Albini TA, Townsend JH, et al. Vision loss after intravitreal injection of autologous "stem cells" for AMD. New England Journal Medicine. 2017;(376):1047-1053.

33. Xu WT, Bian ZY, Fan QM, et al. Human mesenchymal stem cells (hMSCs) target osteosarcoma and promote its growth and pulmonary metastasis. Cancer Lett. 2009;281(1):32-41.

34. Albarenque SM, Zwacka RM, MohrA. Both human and mouse mesenchymal stem cells promote breast cancer metastasis. Stem Cell Res. 2011;7(2):163-171.

35. Ramasamy R, Lam EW, Soeiro I, et al. Mesenchymal stem cells inhibit proliferation and apoptosis of tumor cells: impact on in vivo tumor growth. Leukemia. 2007;21(2):304-310.

36. Sommersall AC. The Amazing Power of STEM CELL NUTRITION: How to Enhance Your Natural Repair System Today. Mississuga Ontario Canada: The Natural Wellness Group; 2013. 349 p.

37. Baraniak PR, Mc Devitt TC. Stem cell paracrine actions and tissue regeneration. Regen Med. 2010;5(1):121-143.

38. Niehans P. Introduction to Cellular Therapy. Pageant Books New York; 1960. 119 p.

39. Yu B, Zhang X, Li X. Exosomes derived from mesenchymal stem cells. Int J Mol Sci. 2014;15(3):4142-4157.

40. Katsuda T, Kosaka N, Takeshita F, et al. The therapeutic potential of 
mesenchymal stem cell-derived extracellular vesicles. Proteomics. 2013;13(10-11):1637-1653.

41. Ren G, Zhang L, Zhao X, et al. Mesenchymal stem cell-mediated immunosuppression occurs via concerted action of chemokines and nitric oxide. Cell Stem Cell. 2008;2(2):141-150.

42. Chang BL, Beer J, Percec I. Platelet-rich plasma: Fact or fantasy? Advances in Cosmetic Surgery. 2018;1(1):193-209.

43. Lubkowska A, Dolegowska B, Banfi G. Growth factor content in PRP and their applicability in medicine. J Biol Regul Homeost Agents. 2012;26(2 Suppl. 1):3S-22S.

44. Shao C, Sima J, Zhang SX, et al. Suppression of corneal neovascularization by PEDF release from human amniotic membranes. Invest Ophthalmol Vis Sci. 2004;45:1758-1762.

45. Faridvan Y, Nozari S, Atashkhoei S, et al. Amniotic membrane extracted proteins protect $\mathrm{H} 9 \mathrm{c} 2$ cardiomyoblasts against hypoxia-induced apoptosis by modulating oxidative stress. Biochemical and Biophysical Communications. 2018;503(3):1335-1341.

46. Martin WJ. Stealth Adapted Viruses; Alternative Cellular Energy (ACE) and KELEA Activated Water. Indiana, USA: Author House; 2014. 321 p.

47. Martin WJ. Complex intracellular inclusions in the brain of a child with a stealth virus encephalopathy. Exp Mol Pathol. 2003;74(3):179-209.

48. Martin WJ. Stealth virus culture pigments: A potential source of cellular energy. Exp Mol Pathol. 2003;74(3):210-223.

49. Martin WJ. Progressive medicine. Exp Mol Pathol. 2005;78(3):218-220.

50. Martin WJ. Etheric biology. Exp Mol Pathol. 2005;78:221-227, 2005.

51. Martin WJ. Deconstructing medicine. The alternative cellular energy pathway. British Journal of Medicine \& Medical Research. 2016;11(8):16.

52. Martin WJ. KELEA: A natural energy that seemingly reduces intermolecular hydrogen bonding in water and other liquids. Open Journal of Biophysics. 2015;5(3):69-79.

53. Martin WJ. Improved efficiency of heat exchange using KELEA activated water. Open Journal of Energy Efficiency. 2015;4:36-43.

54. Martin WJ. KELEA (kinetic energy limiting electrostatic attraction) offers an alternative explanation to existing concepts regarding wave-particle duality, cold fusion and superconductivity. Journal of Modern Physics. 2016;7(15):1995-2007.

55. Martin WJ. Is KELEA [kinetic energy limiting electrostatic attraction) A source of chemical energy? MOJ Bioorganic Inorganic Chemistry. 2017;1(2):00011.

56. Martin WJ. KELEA (kinetic energy limiting electrostatic attraction) can markedly improve the performance of gasoline and diesel fuels in power generation and in transportation. Journal of Transportation Technologies. 2016;6(3):148-154

57. Martin WJ. Is the brain an activator of the alternative cellular energy (ACE) pathway? International Journal of Complementary \& Alternative Medicine. 2015;1(1):00002.

58. Martin WJ. Interactive electric fields can attract KELEA [kinetic energy limiting electrostatic attraction) and can lead to the activation of water. Int J Complement Alt Med. 2015;1(6):00034.

59. Martin WJ. Interacting light paths attract KELEA [kinetic energy limiting electrostatic attraction) and can lead to the activation of water. Open Journal of Biophysics. 2015;5(4):115-121.
60. Martin WJ. The many biological functions of the alternative cellular energy (ACE) pathway. Int J Complement Alt Med. 2017;7(5):00237.

61. Martin WJ. Therapeutic potential of KELEA activated water. Int $J$ Complement Alt Med. 2015;1(1):00001.

62. Martin WJ. KELEA Activation of Water and Other Fluids for Health, Agriculture and Industry. Journal of Water Resource and Protection. 2015;7(16):1331-1344.

63. Martin WJ. Preparing and using KELEA activated water to enhance the alternative cellular energy (ACE) pathway in the therapy of multiple illnesses. Int J Complement Alt Med. 2016;3(1):00059.

64. Martin WJ. Alternative cellular energy pathway therapy using KELEA activated water. Int J Complement Alt Med. 2015;2(2):00051.

65. Dubrov V, Dubrova T, Christner D, et al. Alternative cellular energy based therapy using Enercel ${ }^{\mathrm{TM}}$ in advanced AIDS patients co-infected with tuberculosis and treated in Chernigov, Ukraine. $J$ Hum Virol Retrovirol. 2015;2(6):00061.

66. Martin WJ, Laurent D. Homeopathy as a misnomer for activation of the alternative cellular energy pathway: Evidence for the therapeutic benefits of Enercel in a diverse range of clinical illnesses. Int J Complement Alt Med. 2015;2(1):00045.

67. Martin WJ. Cancer as an insufficiency of cellular energy (ICE) Therapeutic approaches based on enhancing the alternative cellular energy (ACE) pathway. Int J Complement Alt Med. 2016;3(3):00074.

68. Martin WJ. Alternative Cellular Energy Based Therapy of Childhood Diarrhea In Stealth Adapted Viruses; Alternative Cellular Energy (ACE) and KELEA Activated Water. Indiana, USA: Author House; 2014. p. 103-114.

69. Martin WJ. Tissue regeneration without scarring achieved by enhancing the alternative cellular energy (ACE) pathway. Journal of Cosmetics, Dermatological Sciences and Applications. 2017;7(1):82-98.

70. Martin WJ, Stoneburner J. Symptomatic relief of herpetic skin lesions utilizing an energy based approach to healing. Exp Mol Path. 2005;78(2):131-134.

71. Martin WJ, Stoneburner J. Alternative cellular energy (ACE) pathway activation as the mode of action of neutral red dye phototherapy of human viruses. J Hum Virol Retrovirol. 2014;1(4):00019.

72. Martin WJ. Neutral Red Dye/Ultraviolet Light Activation of the Alternative Cellular Energy (ACE) Path way: A Historical Overview and Current Use of Neutral Red Dye Phototherapy of Herpes Simplex Virus infections In Stealth Adapted Viruses; Alternative Cellular Energy (ACE) and KELEA Activated Water. Indiana, USA: Author House; 2014. p. 71-86.

73. Martin WJ. Alternative Cellular Energy (ACE) Pathway Activationas Natural therapy for Autism In Stealth Adapted Viruses; Alternative Cellular Energy (ACE) and KELEA Activated Water. Indiana, USA: Author House; 2014.p. 87-102.

74. Martin WJ. Insufficiency of cellular energy (ICE) may precede neurodegeneration in Alzheimer's disease and be treatable via the alternative cellular energy (ACE) pathway. Advances in Alzheimer's Disease. 2016;6(1):1-12.

75. Martin WJ. Insufficiency of cellular energy (ICE) from the alternative cellular energy (ACE) pathway limiting the specialized functions of neuronal cells. Int J Complement Alt Med. 2017;4(2):00112.

76. Martin WJ. Insufficiency of cellular energy (ICE) in neurons: From electrical hyperactivity to quiescence. Int $J$ Complement Alt Med. 2017;4(3):00118. 
77. Martin WJ. The many biological functions of the alternative cellular energy (ACE) pathway. Int J Complement Alt Med. 2017;7(5):00237.
78. Martin WJ. Is the placebo effect mediated by the alternative cellular energy (ACE) pathway? Complement Alt Med. 2018;11(4):231-233. 